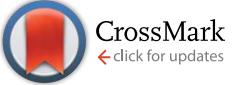

Cite this: RSC Adv., 2017, 7, 10707

Received 5th December 2016 Accepted 23rd January 2017

DOI: 10.1039/c6ra27763b

rsc.li/rsc-advances

\section{Novel application of peptaibiotics derived from Trichoderma sp. for methanogenic suppression and enhanced power generation in microbial fuel cells $\uparrow$}

\begin{abstract}
Ghosh Ray, ${ }^{a}$ Md. T. Noori ${ }^{b}$ and M. M. Ghangrekarc
A major limitation to achieve higher power output from microbial fuel cells (MFC) is the development of competitive environment for substrate utilization offered by methanogenic archaea. This study reveals the comparative electrical performance of MFC, where the enrichment of anodic electrogenic inoculum was done with two low-cost peptaibiotics recovered from Trichoderma sp. Membrane active, poreforming compounds such as the peptaibiotics Trichotoxin A-40 and Alamethicin F-50 were found in $T$. viride extract, whereas, Neoatroviridin (A-D) was retrieved from $T$. atroviride. Upon treatment of mixed anaerobic sewage sludge, used as the inoculum in MFC, respective power densities of $205.40 \mathrm{~mW} \mathrm{~m}^{-2}$ (790 $\mathrm{mA} \mathrm{m}^{-2}$ ) and $306.03 \mathrm{~mW} \mathrm{~m}^{-2}\left(1010 \mathrm{~mA} \mathrm{~m}^{-2}\right)$ were demonstrated by MFCs with significantly high coulombic efficiencies of $48.7 \%$ and $53.5 \%$, respectively. These power densities were 3.4 and 2.4 times higher than that was produced by the control MFC, operated without pre-treating the inoculum with fungal extract. The inhibitory effect on methanogens was sustained even after 15 batch cycles of MFC operation, demonstrating no adverse effect of such peptaibiotics on electrogens, but providing a noncompetitive environment for their growth by effectively inhibiting methanogens and considerably enhancing the power recovery from MFC.
\end{abstract}

\section{Introduction}

The generation of electricity from organic wastes using electrogenic microorganisms in microbial fuel cells (MFC) is one of the breakthrough approaches in bioenergy research, providing an alternative source of electrical energy for charging low power demanding appliances and super-capacitors. Microorganisms in their metabolic processes oxidize soluble as well as colloidal organic matter to produce electrons. Generated electrons travel through a series of respiratory enzymes and reduce protein complexes, which consequently leads to the production of adenosine triphosphate (ATP) as the energy currency by this process. In the anodic chamber of MFC, electrons reduce the anode and are finally transported towards the cathode through an external circuit, where the terminal electron acceptors (TEA) such as oxygen, nitrate, sulphate or any other alternate oxidant exist.

A few bacteria, known as electrogens, encourage exogenous electron transfer by mediators produced by the cells, or

\footnotetext{
${ }^{a}$ P. K. Sinha Center for Bioenergy, Advanced Technology Development Centre, Indian Institute of Technology, Kharagpur 721302, India

${ }^{b}$ Department of Agricultural and Food Engineering, Indian Institute of Technology, Kharagpur, 721 302, India

${ }^{c}$ Department of Civil Engineering, Indian Institute of Technology, Kharagpur 721302, India. E-mail: ghangrekar@civil.iitkgp.ernet.in; Tel: +91-3222-283440

$\dagger$ Electronic supplementary information (ESI) available. See DOI: 10.1039/c6ra27763b
}

externally added metal oxides, to carry over the electrons to the anode to produce current in the MFC. An irreversible coulombic loss occurs in MFC inoculated with mixed anaerobic sewage sludge, as electrogenic bacteria strive to be in close competition with methanogenic bacteria for utilization of the substrate. Hence, various amounts of methane production was observed in many MFCs, due to the similar growth conditions of methanogens and exo-electrogens. Hydrogenotrophic methanogenesis caused $15-25 \%$ of the substrate-loss in continuous glucose-fed MFC; whereas, in the slow growing process of acetoclastic methanogenesis, over $50 \%$ of substrate was converted to methane during a long-term operation when the organic loading exceeded the optimum value for electrogens., ${ }^{\mathbf{1 2}}$

In order to improve the power recovered from MFC, suppression of electron loss through substrate utilization by methanogens is required. Methanogens, being obligatory anaerobes, are extremely sensitive to oxygen exposure. Several studies have reported short-term methanogenic suppression in MFC when exposed to environmental stresses such as oxygen, low temperature, low $\mathrm{pH}$, chemical inhibitors and variation in external resistances, ${ }^{\mathbf{1 , 3}, \mathbf{4}}$ or with an improved MFC configuration. ${ }^{5}$ Specific methanogenic inhibition by using 2-bromoethanesulfonate (BES), 2-chloroethanesulfonate (CES) and 2mercaptoethane-sulfonate (MES) involves the inhibition of methyl group transfer by coenzyme $\mathrm{M}$, dominantly found in all methanogens, at the terminal reductive step of methane biosynthesis. $^{6}$ 
Lethal invasion of halogenated aliphatic hydrocarbons such as chloroform $\left(\mathrm{CHCl}_{3}\right)$ at low concentration $(100 \mu \mathrm{M})$ and fluoro-acetate $\left(\mathrm{FCH}_{2} \mathrm{COO}^{-}\right)$has also been reported, where the inhibition of methyl-coenzyme $\mathrm{M}$ reductase during acetoclastic and hydrogenotrophic methane production was observed by the former; whereas, the latter was found to suppress acetoclastic methanogenesis by inhibiting the activity of acotinase in the tricarboxylic acid (TCA) cycle. ${ }^{3}$ Bio-agents, such as coenzymes lumazine, ${ }^{7}$ medium (lauric acid) and long chain fatty acids (caprylic, capric, myristic and hexa-decatrienoic acid) and alamethicin are highly specific inhibitors of methanogenesis, where the inhibition of Gram-positive methanogenic bacteria occurs upon contact via absorption and disruption of cell membranes; ${ }^{\mathbf{8}, 9}$ substrate loss due to the activity of methanogenic bacteria in bio-electrochemical systems reduces the cumulative recovery of electrons and lowers the coulombic efficiency (CE). Studies on methanogenic suppression in MFC showed an increase in $\mathrm{CE}$ up to $70 \%$ using $\mathrm{BES}^{\mathbf{1 0}}$ and in microbial electrolysis cells (MEC), using antibiotics such as neomycin sulphate, BES, CES and 8-aza-hypoxanthine as methanogenic suppressor, the CE was reported as 59\%, 36\%, $33 \%$ and $63 \%$, respectively. ${ }^{11}$ Few studies reported the degradation of sulfamethoxazole antibiotic (200 ppm) in MFC, where higher electrogenic bio-film was observed to be in the active state, demonstrating simultaneous power generation and degradation of antimicrobial agents in MFC. ${ }^{12,13}$

The selective antimicrobial activity of peptaibiotics, a wide array of secondary metabolites derived from Trichoderma sp., has been studied in the past few decades. ${ }^{\mathbf{1 4}}$ The sub-family peptaibols (MW 500-2200 Da) are comprised of approximately 7-20 residues of membrane active hydrophobic amino acids, characterized by the presence of $\alpha$-amino-iso-butyric acid (Aib), a non-proteinogenic amino acid and an amino alcohol at the C-terminal of the poly-peptide chain. The membrane active peptaibols have the unique ability for pore formation through the bacterial cell membrane, leading to the loss of osmotic balance and cytolysis. ${ }^{15-17}$ Gram-positive bacteria and fungi were observed to be sensitive towards peptaibols, but seem ineffective against Gram-negative bacteria. ${ }^{\mathbf{1 8}}$ For Gram-negative bacteria, the outer envelope is composed of lipopolysaccharides (LPS), which provide intrinsic resistance toward the invasion of toxic bioactive compounds. ${ }^{19,20}$ The cell envelope of Gram-negative bacteria acts as an impermeable barrier, allowing the large molecules and hydrophobic toxic compounds (biocides and antibiotics) to penetrate 50-100 times slower than Gram-positive bacteria (except mycobacteria), which lack the outer envelope. ${ }^{21-23}$

Typical and common methanogenic bacteria (such as Methanobacterium sp., Methanosarcina sp. and Methanobrevibactor sp.), found in mesophilic anaerobic sewage sludge, stain Grampositive and belong to the genera Archaea. This prokaryotic archaea cell wall is devoid of LPS and composed of pseudomurein, with a similar characteristic structure to peptaidoglycans. $^{24,25}$ The outer membrane permeability of this genus is likely to be greater for small hydrophobic bioactive compounds, subsequently causing cellular lysis. ${ }^{21}$ It was hypothesized in the present study that such bioactive compounds exhibit a specific selective effect for electrogens by suppressing the population of methanogens. Therefore, this research aimed to extract costeffective secondary metabolites from Trichoderma viride and Trichoderma atroviride, which were examined for their potential to suppress the methanogenic archaea from the mixed anaerobic sewage sludge used as inoculum in MFC, in order to increase the coulombic yield and power recovery.

\section{Materials and methods}

\subsection{Screening and identification of peptaibiotics from Trichoderma sp.}

2.1.1. Growth of fungi - strain maintenance and preparation. Two fungal strains, T. viride (NCIM Accession no.: 1051) from National Chemical Laboratory, Pune, India and T. atroviride (MTCC 9641) from Microbial Type Culture Collection and Gene Bank, Chandigarh, India, were procured as lyophilized cultures and grown in potato dextrose (PD) agar media for 7 days at $30{ }^{\circ} \mathrm{C}$. Fungal cultivation was performed in six $500 \mathrm{~mL}$ culture flasks, each containing $300 \mathrm{~mL}$ of PD broth media and having the composition of potato $-200 \mathrm{~g} \mathrm{~L}^{-1}$, dextrose $-20 \mathrm{~g}$ $\mathrm{L}^{-1}$, and $\mathrm{pH}$ adjusted to $5.2 \pm 0.2$, and inoculated with twelve 8 $\mathrm{mm}$ fungal agar plugs $\left(6.08 \mathrm{~cm}^{2}\right)$. The flasks were maintained at $30{ }^{\circ} \mathrm{C}$ for 5 days on a rotary shaker $(120 \mathrm{rpm})$. The resultant biomass was centrifuged, separated from the suspension and washed thrice with distilled water to remove the remaining media.

2.1.2. Screening and extraction. Mechanical disruption of the fungal cell wall was done by homogenization (REMI Motors Ltd., India) at $8000 \mathrm{rpm}$ for $5 \mathrm{~min}$, followed by intermittent cooling in an ice-bath. Solid-phase, two-solvent extraction of metabolites and small peptides from the fungal slurry was done in $80 \%$ aqueous acetone (media to solvent ratio of $2.4: 1$ ) overnight with shaking. The acetone-extract was filtered, discarding the biomass, and the acetone layer was concentrated in vacuo at $40{ }^{\circ} \mathrm{C}$ using a Büchi Rotavapor R-210 (Büchi Labortechnik AG, Flawil, Switzerland). The fungal extract was further exposed to ethyl acetate extraction thrice (media to solvent ratio of $4: 1$ ) in a separatory funnel. The organic top layer of ethyl acetate containing the fungal bio-active compounds and peptide antibiotics were separated and the solvent was again evaporated under vacuum. The crude extract was further washed with hexane and finally dried to a non-flowing consistency in a water bath, set at $40{ }^{\circ} \mathrm{C}$. The crude extracts were obtained from both strains and used as a source of secondary metabolites/peptaibiotics for further study of methanogenic suppression in MFC.

2.1.3. Chromatographic separation and detection by mass spectrometry. The crude fungal extracts were re-suspended in acetonitrile (ACN - LC gradient grade) to the final concentration of $5 \mathrm{mg} \mathrm{mL} \mathrm{m}^{-1}$. The chromatographic separation was carried out at $25{ }^{\circ} \mathrm{C}$ by reverse phase-high performance liquid chromatography (RP-HPLC), in a HPLC series 1100 (Agilent Technologies, Waldbronn, Germany), with acetonitrile - Milli-Q water with $0.1 \%(\mathrm{v} / \mathrm{v})$ TFA elution and using a $100 \mathrm{~mm} \times 4.6 \mathrm{~mm}$ Eclipse Plus C18 column with $95 \AA$ pore size (Agilent, USA). The concentration gradient started with $10 \%(\mathrm{v} / \mathrm{v}) \mathrm{ACN}$ and then 
increased to $80 \%(\mathrm{v} / \mathrm{v})$ ACN within $60 \mathrm{~min}$, and finished at $80 \%$ $(\mathrm{v} / \mathrm{v}) \mathrm{ACN}$. Detection was done at $216 \mathrm{~nm}$ and the observed peaks were collected for further identification.

Sequence identification of the peptide present in the crude extracts was done by mass spectrometric (MS) analysis in the Central Research Facility, Indian Institute of Technology, Kharagpur, India, by matrix-assisted laser desorption/ ionization (MALDI) time of flight (TOF)/TOF (UltrafleXtreme, Bruker Daltonics, Bremen, Germany) mass spectrometer operated in reflectron mode and calibrated with Protein Calibration Standard, Bruker. The fractions collected from HPLC were mixed with a 4-hydroxycinnamic acid matrix and the mixture was spotted on the MALDI plate and dried at room temperature. Spectra were acquired in the mass range of $600-2500 \mathrm{~m} / \mathrm{z}$ in positive ion mode. After analysing, the MS spectra and the MS/ MS spectra were obtained by LIFT fragmentation, selecting the precursor ions. ${ }^{26}$ Peptide mass fingerprinting was obtained according to Stoppacher et al. ${ }^{27}$ referring to "The Comprehensive Peptaibiotics Database” project (containing 1351 peptaibiotics entries).

2.1.4. Specific methanogenic activity test of the inoculum used in MFC. Mixed anaerobic sewage sludge was used as inoculum in MFCs. Thus, the major challenge was to enhance the aggregation of electrogenic bacteria in the anodic chamber of MFC and suppress the methanogenic bacterial activity in order to reduce the competition for substrates. Sludge inoculum treated with extracted fungal isolates was tested for specific methanogenic activity (SMA). The experiment was performed in three serum bottles containing sludge with fixed concentration of initial volatile suspended solids (VSS) and named the control. The serum bottle containing untreated sludge was treated with $T$. viride extract and named Test 1-SMA; similarly, the serum bottle containing sludge treated with $T$. atroviride extract was named Test 2-SMA. Acetate as the carbon source was used as feed with concentration of $1 \mathrm{~g} \mathrm{COD} \mathrm{L}^{-1}$ and serum bottles were mounted with gas collection units. The produced methane was collected by the liquid displacement method, using $5 \% \mathrm{NaOH}$, registered through a graduated trap cylinder and SMA of the sludge treated with fungal extracts and untreated anaerobic sewage sludge was calculated according to eqn. (1). ${ }^{28}$ were fabricated, namely Control-MFC, without electrode modification and no inoculum pre-treatment; Cat-MFC, having $\mathrm{V}_{2} \mathrm{O}_{5^{-}}$ MF catalysed cathode and GO-PTFE modified anode; TV-MFC, having the $\mathrm{V}_{2} \mathrm{O}_{5}$-MF catalysed cathode, GO-PTFE modified anode and mixed anaerobic sewage sludge as anodic inoculum pre-treated with the crude extract of T. viride; TA-MFC, with $\mathrm{V}_{2} \mathrm{O}_{5}$-MF catalysed cathode, GO-PTFE modified anode and anodic inoculum pre-treated with crude extract of $T$. atroviride. These MFCs had a rectangular shape made of transparent acrylic (25 mm width) and anodic/cathodic chamber working volume of $95 \mathrm{~cm}^{3}$. Electrodes were connected through concealed copper wire and potentials were measured with respect to $100 \Omega$ external load.

The anodic chambers of Control-MFC and Cat-MFC were inoculated with untreated mixed anaerobic sewage sludge (24.22 $\mathrm{g}$ of VSS L ${ }^{-1}$ ), collected from the bottom of a septic tank having a mixed bacterial consortium and without giving any pre-treatment. The inoculum of TV-MFC and TA-MFC were prepared by treating the sewage sludge overnight, shaking with fungal crude extract of $T$. viride and $T$. atroviride with a dosage of $200 \mu \mathrm{g} \mathrm{mg}^{-1}$ of sludge VSS, respectively. Analysis of MFC containing freshly prepared synthetic wastewater with acetate as a carbon source to maintain $2 \mathrm{~g}$ of chemical oxygen demand (COD) per L and pH adjusted to 7.2 with necessary nutrients. ${ }^{9}$ Total COD and solid analysis was monitored according to Standard Methods. ${ }^{31}$

All MFCs were operated under identical conditions in order to compare the effect of methanogen suppression and subsequent electrical behaviour at $35 \pm 3{ }^{\circ} \mathrm{C}$ operating temperature; $72 \mathrm{~h}$ of the fed-batch cycle of operation was adopted, as most of the acetate was consumed within that time. During the steadystate of operation, polarization curves were obtained to determine the maximum power generation. After 8 complete batch cycles, TV-MFC and TA-MFC were again spiked with their respective fungal extracts and electrical behaviour was further monitored.

2.2.2. Data acquisition and electrochemical analysis. To maintain the electro-neutrality, protons, generated due to acetate oxidation, migrated from the anodic chamber to the cathodic chamber to participate in the cathodic reduction. Current $(I)$ generated from the MFCs was calculated for a given

$$
\mathrm{SMA}=\frac{\text { methane production }\left(\mathrm{g} \mathrm{COD} \mathrm{d}^{-1}\right)}{\text { active volume of reactor }(1) \times \text { biomass conc. of SMA test reactor }\left(\mathrm{g} \mathrm{VSS}^{-1}\right)}
$$

\subsection{Operation and performance monitoring of microbial fuel cells}

2.2.1. MFC design, fabrication and operation. Graphene oxide/polytetrafluoroethylene (GO-PTFE) modified composite carbon felt electrodes were prepared as the anode, ${ }^{29}$ and the cathode was modified with vanadium pentoxide micro-flowers $\left(\mathrm{V}_{2} \mathrm{O}_{5}-\mathrm{MF}\right){ }^{30}$ The detailed method of electrode preparation is described in the ESI. $\dagger$ Four double-chamber microbial fuel cells resistance $(R)$ from the acquired voltage $\left(V_{\text {cell }}\right)$ by $I=V_{\text {cell }} / R$. Voltage was measured using a data acquisition/switch unit (Agilent Technologies, Malaysia) attached to a computer system. Coulombic efficiency $\left(C_{\mathrm{E}}\right)$ or the fraction of recovered electrons was estimated as per eqn (2):

$$
C_{\mathrm{E}}=\frac{M_{\mathrm{s}} \int_{0}^{t} I \mathrm{~d} t}{F b_{\mathrm{es}} V_{\mathrm{An}} \Delta \mathrm{COD}}
$$


where, $M_{\mathrm{s}}$ is the molecular weight of oxygen $\left(32 \mathrm{~g} \mathrm{~mol}^{-1}\right), \Delta \mathrm{COD}$ is the change in substrate concentration over a batch cycle $\left(\mathrm{g} \mathrm{L}^{-1}\right)$, $V_{\text {An }}$ is the anodic volume $(\mathrm{L}), F$ is Faraday's constant $(96485 \mathrm{C}$ $\mathrm{mol}^{-1} \mathrm{e}^{-}$) and $b_{\text {es }}$ is the generated electrons during each mole of substrate oxidation ( $4 \mathrm{~mol} \mathrm{e}^{-}$per mole COD). The anodic half-cell potential was measured by placing a $\mathrm{Ag} / \mathrm{AgCl}$ reference electrode (CH Instruments, Inc., RE-5B; +0.197 V vs. a standard hydrogen electrode, SHE) inside the anodic compartment. The polarization study was done by monitoring the voltage output from MFCs at various external resistances, ranging from $30000 \Omega$ to $5 \Omega$, to obtain the relationship between voltage and current. The corresponding voltage at each resistance was measured at an interval of 10-15 min to get a stable reading. Power $(P)$ was calculated by $P=I$ $\times V_{\text {cell }}$ and normalized by the geometrical surface area of the anode $\left(30 \mathrm{~cm}^{2}\right)$. To understand the metabolic activity of mixed microbial consortia inside the anodic chamber and the effect of methanogenic suppression by fungal peptaibiotics, the anodic chamber head-space biogas analysis was done using gas chromatography (Perkin-Elmer Auto System XL GC, USA) with a thermal conductivity detector (TCD). ${ }^{32}$ Detection of methane and carbon-dioxide was done in a Porapak-Q column using nitrogen as the carrier gas and keeping the oven temperature at $80{ }^{\circ} \mathrm{C}$.

Electrochemical studies were performed in situ on the anodic half-cells of MFCs using a potentiostat (AUTO LAB AUT58696, Metrohm), connected to a computer-based analyzer. Cyclic voltammetry $(\mathrm{CV})$ is one of the versatile techniques that allows one to understand the mechanics of the electron transfer processes in MFC during the oxidation of organic matter present in wastewater at the anode-biofilm interface via extracellular redox-active mediators, their charge-discharge pattern, corresponding current outputs and the nature of biofilm grown over the electrode surface. ${ }^{33,34} \mathrm{CV}$ was performed using a three-electrode assembly, with the anode as the working electrode (WE), a platinum wire as the counter electrode and a $\mathrm{Ag} / \mathrm{AgCl}$ reference electrode (RE). The potential between the $\mathrm{WE}$ and the RE was varied from -1 to $+1 \mathrm{~V}$ at an applied scan rate of $10 \mathrm{mV} \mathrm{s}^{-1}$ and the corresponding current generated between the WE and the counter electrode was measured, avoiding contact between the electrodes. In our case, while working with mixed anaerobic sludge inoculum, the bacteria attached to the anodic bio-film were expected to produce redox mediators to enable electron shuttling. The membrane bound electron transfer, as well as the transfer through soluble redox mediators were studied when a fixed scan rate of $10 \mathrm{mV} \mathrm{s}^{-1}$ was maintained for all MFCs during CV analysis. ${ }^{35,36}$ Electrochemical impedance spectroscopy (EIS) measurements were performed with frequency ranging from $100 \mathrm{kHz}$ to $100 \mathrm{MHz}$. An electrical equivalent circuit was designed in order to achieve the best fit for the simulation of observed experimental EIS data. The fitting and EIS data analysis was done by EC-Lab V11.01 software.

\section{Results and discussion}

\subsection{Analysis and identification of peptaibiotics}

Five fractions from $T$. viride and six fractions from $T$. atroviride were detected in RP-HPLC (Fig. 1), showing their hydrophobic

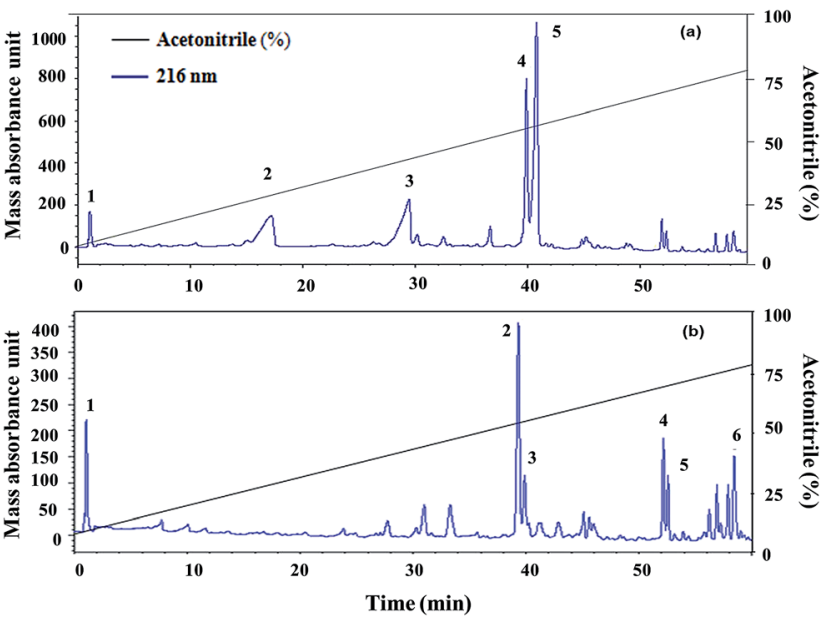

Fig. 1 (a) Trichoderma viride and (b) Trichoderma atroviride extract chromatography (RP-HPLC): detection at $216 \mathrm{~nm}$ in a gradient of acetonitrile and Milli-Q water (with $0.1 \%$ TCA).

profile and these were collected for peptide mass fingerprinting. Peptaibiotics detection at retention time of $40.761 \mathrm{~min}$ for fraction 4 of the $T$. viride extract revealed the presence of two prominent ions with $m / z 1018.678$ and 1975.498. Further fragmentation of the ion with $\mathrm{m} / \mathrm{z} 1018.678$ rendered a partial interpretation of the sequence containing six amino acid residues (Ac-Aib-Gly-Aib-Leu-Aib-Glu) with the characteristic Ac group of peptaibiotics at the N-terminus (Fig. 2a) of the polypeptide chain, which was identified as part of Trichotoxins A-40. ${ }^{37}$ Upon further fragmentation of the ion with $m / z$ 1975.498, a partial sequence of fourteen amino acid residues (Gln-Aib-Val-Aib-Gly-Leu-Aib-Pro-Val-Aib-Aib-Gln-GlnPhe-ol) was identified as Alamethicin F-50 (Fig. 2b). ${ }^{38}$

In RP-HPLC, six fractions were obtained for the $T$. atroviride extract in which peptaibiotics were detected only for three fractions (2, 3 and 4). In fraction 4, the most prominent ions were those with $\mathrm{m} / \mathrm{z} 1801.203$ and 1187.143. Fragmentation and interpretation of the latter was not possible, due to analytical limitations; however, on analyzing the ion with $\mathrm{m} / \mathrm{z}$ of 1801.203, a partial sequence was observed (Aib-Leu-Aib-Gly-Iva-Aib-ProLeu-Aib-Aib-Glu-Leu-ol) (Fig. 3), suggesting the presence of Trichoderma peptaibiotic, having an 18-residue amino acid structure. As reported by Oh et al., the obtained partial sequence was similar to the reported sequence for isolated novel antibiotic peptaibols Neoatroviridin (A-D) from T. atroviride ${ }^{39}$ Fraction 2 and 3 both showed the characteristic peptaibiotic sequence of the most prominent ion with $\mathrm{m} / \mathrm{z} 1410.5$ (refer to ESI, Fig. $\mathrm{S} 1$ and $\mathrm{S} 2 \dagger$ ).

\subsection{Performance of MFCs using peptaibiotic pre-treated anodic inoculum}

Crude extracts, derived from Trichoderma sp. and enriched with peptaibiotics, were selected as methanogen inhibitors in this study. The methanogen inhibition effect enriched the abundance of electrochemically active bacteria in the anodic 


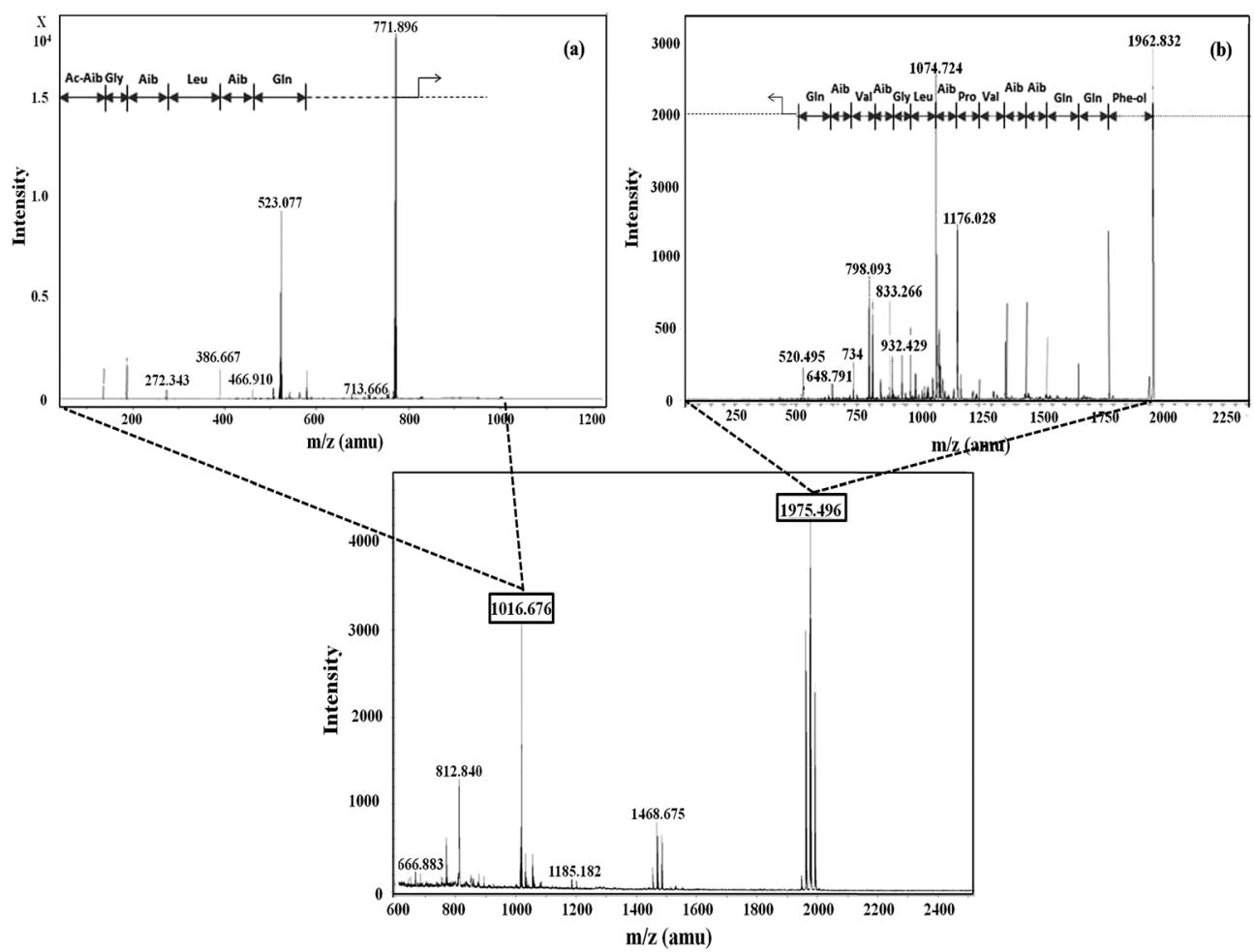

Fig. 2 Mass spectrometric profile of T. viride extract for fraction 4 (bottom); fragmentation of ions (MS/MS) with $\mathrm{m} / \mathrm{z} 1016.6$ (a) and 1975.4 (b).

chamber of TV-MFC and TA-MFC, which was exemplified by their electrical performances and compared with Control-MFC. After around 20 days of operation, the MFCs started exhibiting reproducible cycles of current generation, which might be due to the activity of matured biofilm grown over the anode surface during this start-up period.

Significant differences in working potentials were observed during the stable cycles of MFC operation: $145 \pm 15 \mathrm{mV}$ for Control-MFC, $225 \pm 18 \mathrm{mV}$ for Cat-MFC, $254 \pm 20 \mathrm{mV}$ for TVMFC and $305 \pm 15 \mathrm{mV}$ for TA-MFC, over $100 \Omega$ of external resistance. Operating voltage data for each cycle operation has been depicted in ESI (Fig. S3†). The operating potential of CatMFC was $295 \pm 15 \mathrm{mV}$, which was significantly high, during the initial two weeks of operation; this slowly decreased due to the dominance of methanogenesis over electrogenesis with the prolonged operational period. Even in TV-MFC, a slight drop in operating potential from $286 \pm 14 \mathrm{mV}$ to $254 \pm 20 \mathrm{mV}$ was observed after achieving the steady state because of the possibility of the reoccurrence of methanogens from the pre-treated mixed anaerobic sludge used as inoculum; this voltage drop was not observed in case of TA-MFC. Distinctly improved performance was noted due to the dominance of electrogens in the anodic biofilm, where inoculum was pre-treated with peptaibiotics, generating substantially higher power densities. An increase in the effective surface area by the GO-PTFE modified anode encouraged higher aggregation of biofilm over the anode surface, consequently generating $158.41 \mathrm{~mW} \mathrm{~m}^{-2}(726.67 \mathrm{~mA}$ $\mathrm{m}^{-2}, 22.94 \mathrm{~A} \mathrm{~m}^{-3}$ ) of power density by Cat-MFC (Fig. $4 \mathrm{~b}$ ). Maximum power densities of $306.03 \mathrm{~mW} \mathrm{~m}^{-2}\left(1010 \mathrm{~mA} \mathrm{~m}{ }^{-2}\right.$, $\left.31.89 \mathrm{~A} \mathrm{~m}^{-3}\right)$ for TA-MFC and $205.40 \mathrm{~mW} \mathrm{~m}^{-2}\left(790 \mathrm{~mA} \mathrm{~m}{ }^{-2}\right.$, $27.54 \mathrm{~A} \mathrm{~m}^{-3}$ ) for TV-MFC were obtained, which were 3.4 and 2.4 times higher, respectively, than the Control-MFC, which produced a power density of $86.68 \mathrm{~mW} \mathrm{~m}^{-2}\left(438.89 \mathrm{~mA} \mathrm{~m}^{-2}\right.$, $\left.13.43 \mathrm{~A} \mathrm{~m}^{-3}\right)$. The highest power density of $306.03 \mathrm{~mW} \mathrm{~m}^{-2}$ $\left(9.68 \mathrm{~W} \mathrm{~m}^{-3}\right)$ was demonstrated by TA-MFC, compared to previous research, where a power density of $40 \mathrm{~mW} \mathrm{~m}{ }^{-2}$ was obtained using BES, and a power density of $229.68 \mathrm{~mW} \mathrm{~m}^{-2}$ was reported while using Chaetoceros for the pre-treatment of mixed anaerobic sludge used as anodic inoculum..$^{10,40}$

The whole cell internal resistance of MFCs after 6 weeks of operation was obtained by suitably selecting the near linear portion of the polarization curve. ${ }^{41}$ The lowest whole cell resistance of $87.9 \Omega$ was obtained for TA-MFC, followed by $98.5 \Omega$ and 106.1 $\Omega$ for Cat-MFC and TV-MFC, respectively, with considerably higher internal resistance of $155.4 \Omega$ for ControlMFC (Fig. 4a). Resistive elements for the anodic half-cell were also measured by EIS analysis in order to compare the performance of MFCs under varied conditions of anodic inoculum and have been reported in the latter part of the discussion. No significant effect on power generation was observed when TVMFC and TA-MFC were once again spiked with their respective fungal extracts after eight weeks of operation.

\subsubsection{Electrochemical studies}

3.2.1.1. Cyclic voltammetry. Cyclic voltammetry was used for the characterization of the biological fuel-cell systems, where the reversible electrochemical activities and the propensity for biofilm growth over the anode surface are responsible for electron shuttling in the form of current peaks. The overall 


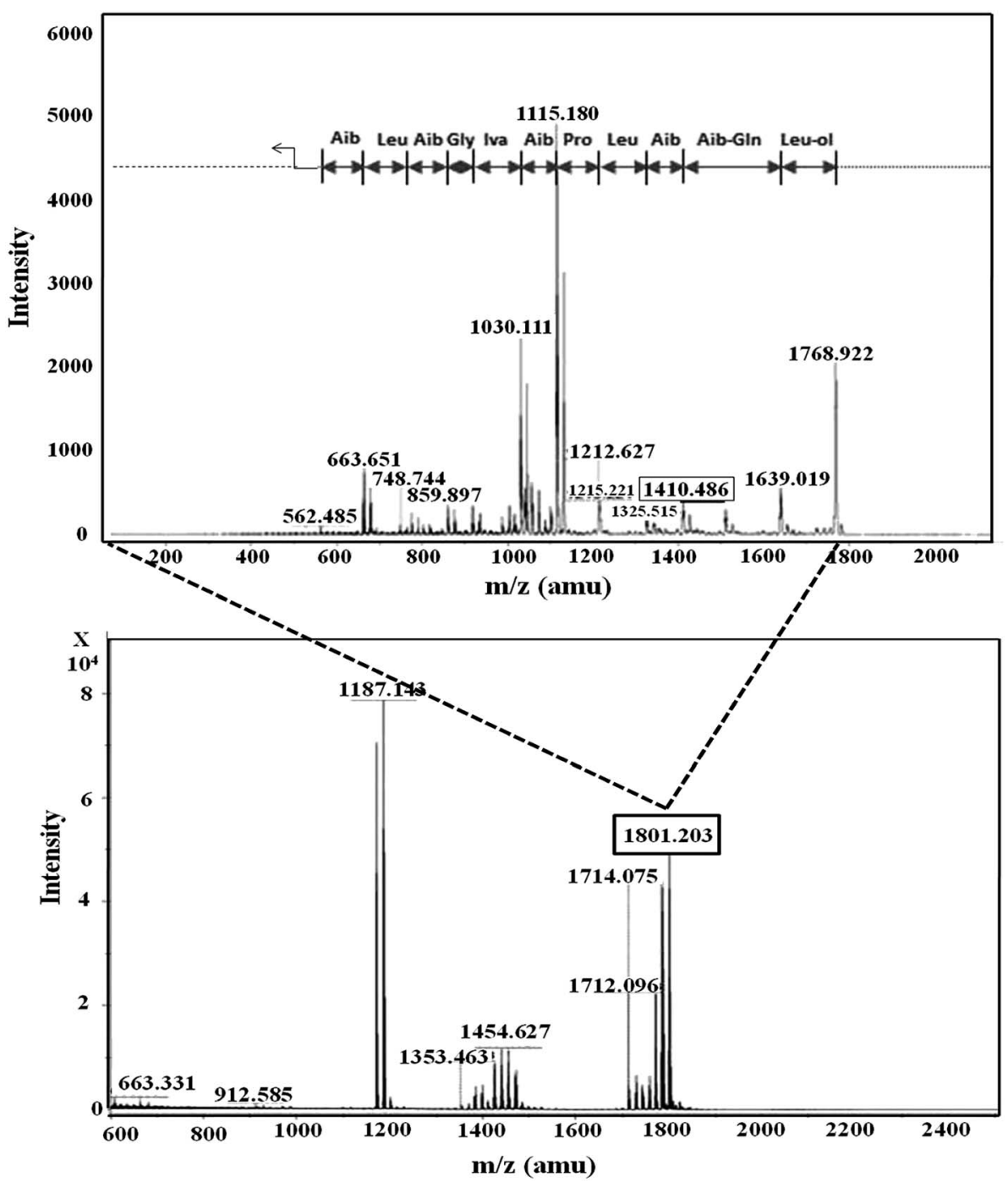

Fig. 3 Mass spectrometric profile of T. atroviride extract for fraction 4 (bottom); fragmentation of ions (MS/MS) with $\mathrm{m} / \mathrm{z} 1801.203$ (top).

impedance of the anodic half-cell was also measured by electrochemical impedance spectroscopy (EIS) in order to understand the effect of modification in anodic inoculum, their charge transfer properties and the contribution of different internal resistances to the anodic impedance of fuel cells.

In order to analyze the biofilm based extra-cellular electron transfer (EET) of electrochemically active microbial consortia, the redox potentials of soluble redox active components were determined by $\mathrm{CV}$. The analysis was performed under the stable-stage turnover conditions of acetate, depicting weak oxidation/reduction peaks in the voltammograms (Fig. 5a). It is important to mention that all three voltammograms exhibited the broader ambit of output current associated with the oxidation of redox-active electron mediators, under the ample supplementation of the electron donor (acetate in the present case), compared to the voltammogram obtained for controlMFC. Due to the higher level of electrogenic bio-film growth of peptaibiotic pre-treated anodic inoculum over the anode surface, the electrical charge holding capacity of the bio-film was also increased, which consequently demonstrated higher capacity current. The proficiency of MFC depends on substrate diffusion over the active biofilm and its ability to produce redox active complexes by controlling the electron transfer processes in order to harvest power to a greater extent.

Two weak oxidation peaks of $4.9 \mathrm{~mA}$ and $5.7 \mathrm{~mA}$ were found during the $\mathrm{CV}$ test for TA-MFC at $-550 \mathrm{mV}$ (vs. $\mathrm{Ag} / \mathrm{AgCl})$ and $-230 \mathrm{mV}$ (vs. $\mathrm{Ag} / \mathrm{AgCl}$ ), and a corresponding single reduction peak of $-4.3 \mathrm{~mA}$ was detected at almost $47 \mathrm{mV}$. In the case of TV-MFC and Cat-MFC, the oxidative current peaks consistently appeared at almost the same potential of $-370 \mathrm{mV}$, with peak current value of $-3.6 \mathrm{~mA}$ and $-4.7 \mathrm{~mA}$, respectively. In addition, TA-MFC delivered higher redox current followed by CatMFC, TV-MFC, and Control-MFC (Fig. 5a), which further confirms the enhanced anode kinetics in TA-MFC due to the distinguished effect of $T$. atroviride peptaibiotics on methanogenic suppression. Moreover, the GO-PTFE modification on the anode surface facilitated a large number of reaction sites for oxidation and can act as the improved final electron acceptor for 

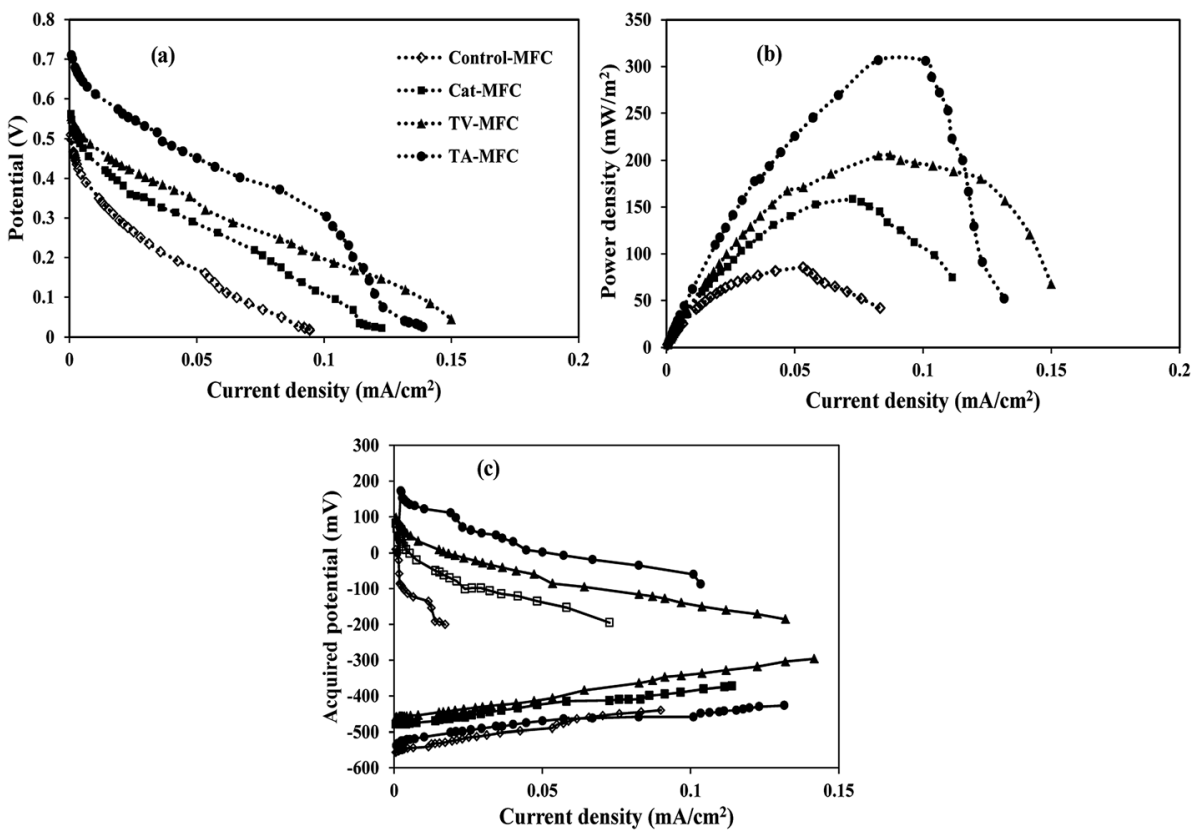

Fig. 4 (a) Cell polarization, (b) power density and (c) half-cell potentials vs. current curves of four MFCs.
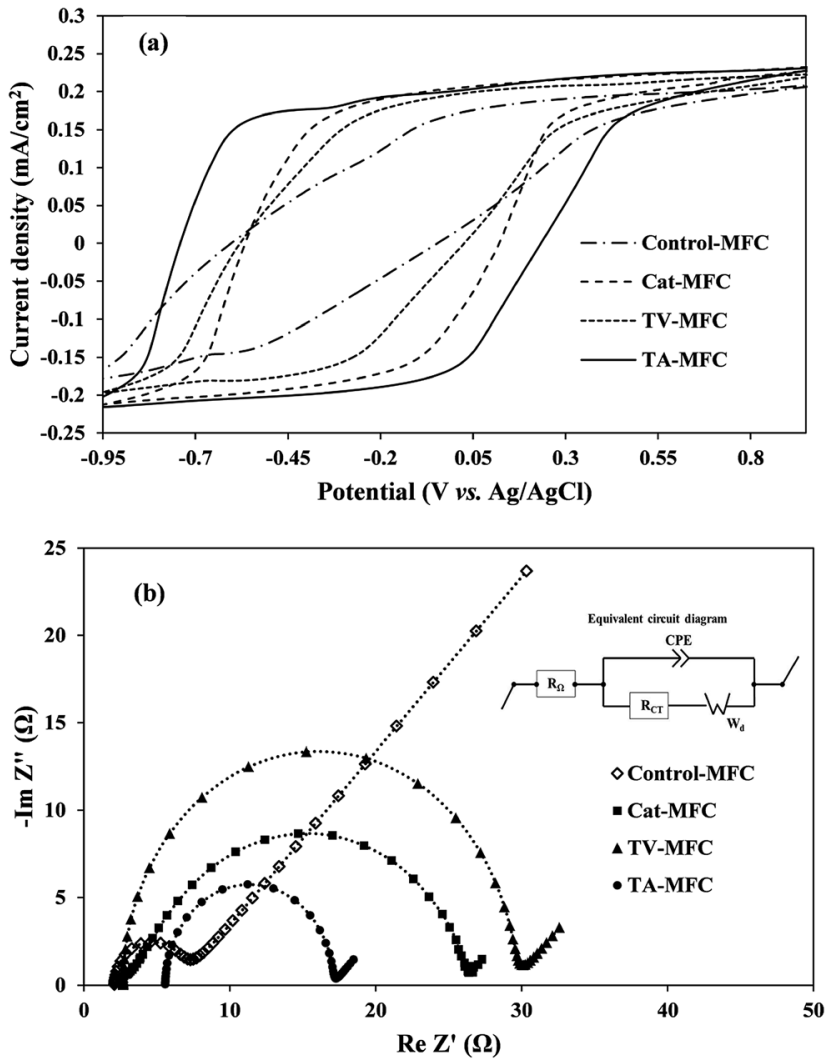

Fig. 5 Electrochemical characteristics of the anode: (a) cyclic voltammogram recorded at a scan rate of $10 \mathrm{mV} \mathrm{s}^{-1}$ in the presence of acetate (turnover condition); (b) Nyquist plot continuous line with simulated data, and discontinuous line with experimental data (week 6) for the analysis of impedance elements (inset - equivalent circuit for EIS data simulation). the respiratory electron transfer in MFCs, thereby enhancing the redox current. ${ }^{42,43}$ However, the voltammogram associated with Control-MFC was unable to reveal any redox current peak and showed significantly lower redox activities (redox current) as compared to the other MFCs with modified anodes and inoculum treated with either T. atroviride or T. viride peptaibiotics, indicating limited charge holding capacity of aged and fully developed biofilm over a long incubation time for ControlMFC. ${ }^{33}$

3.2.1.2. Electrochemical impedance spectroscopy. EIS is a steady-state, non-destructive electrochemical technique, which facilitates the critical understanding of the dynamics of bound or mobile charges in the bulk and electrode/biofilm interface region, the electrochemical properties of redox mediators and the individual components of internal impedance in MFCs. Complex Nyquist impedance spectra were recorded for the anodic chamber with biofilm and analyte after 1 week and 6 weeks of operation in order to understand the effect of the operational time-period on the performance of MFC. Impedance elements for all studies were derived by fitting the EIS experimental data on the electrical equivalent circuit (Fig. $5 b$ ). The major elements of the equivalent circuit that significantly affect the performance of MFCs are as follows: (i) internal ohmic resistance $\left(R_{\Omega}\right)$, which represents the resistance of analyte and membrane towards proton transfer from the anode to the cathode; (ii) charge transfer resistance of electrode and microbially synthesized mediators $\left(R_{\mathrm{CT}}\right)$, which is inversely proportional to the chemical reaction rate at the anode and is represented by the charge-transfer limited semi-circular part at the high to mid frequency range of the impedance spectra; (iii) constant phase element (CPE) corresponding to the electrical double layer pseudo capacitance of the anode/analyte interface. Under the mixed kinetics and charge transfer control, the 
impedance spectrum typically includes the Warburg diffusionlimited straight line ( $W_{\mathrm{d}}$, with $45^{\circ}$ slope) at low frequency. The fitted equivalent circuit appears to be the Randle type circuit, where the Faradaic impedance (combined series effect of charge transfer and diffusion resistance) and double layer capacitance are in parallel connection.

The impedance spectrum obtained after 1 week of operation (provided in ESI, Fig. S4 $\dagger$ ) showed comparatively higher solution resistance $\left(R_{\Omega}\right)$ for TA-MFC and TV-MFC, which might be due to the leaching effect of hydrophilic pigments from peptaibiotics, increasing the overall resistance of the anodic chamber. However, in subsequent cycles of operation, the pigments were washed out with MFC effluent. For TV-MFC and TA-MFC the ohmic resistance was decreased over the experimental period, suggesting lower resistance of analyte and membrane for proton passage; whereas, $R_{\Omega}$ was increased for Control-MFC and Cat-MFC (Table 1). The lowest $R_{\mathrm{CT}}$ for TA-MFC indicates a higher rate of electrochemical reaction in the anode/ analyte interface, reducing the activation barrier and enhancing the acquired power density, which might have facilitated the growth of electrogenic bacteria (except Control-MFC). $R_{\mathrm{CT}}$ can be significantly decreased if the concentration of the redoxactive mediators increases in the analyte. ${ }^{34,44}$ Synthesis of such mediators depends on the availability of electro-active bacteria, where their growth directly depends on the presence of consumable substrate in the analyte and the competitive environment of the mixed consortium. The decrease in the magnitude of the double layer capacitance (CPE) can be correlated to biofilm formation over the anode/analyte interface, due to which the hydrophilicity of the anode increased with enriched electrogenic culture under peptaibiotic pre-treated inoculum as observed from EIS data from the first week and sixth week of operation of MFC. This can be attributed to the conduction of more electrons through the biofilm. A substantial decrease in

Table 1 Impedance elements of the equivalent circuit - electrochemical behaviour of MFCs

\begin{tabular}{lcccc}
\hline Type & $R_{\Omega}(\Omega)$ & $R_{\mathrm{CT}}(\Omega)$ & $W\left(\Omega \mathrm{S}^{-1 / 2}\right)$ & $\mathrm{CPE}(\mu$ \\
\hline Week 1 & & & & \\
Control-MFC $^{a}$ & 1.78 & 2.78 & - & 91.10 \\
Cat-MFC $^{b}$ & 2.14 & 7.34 & 3.45 & 53.50 \\
TV-MFC $^{c}$ & 17.20 & 39.50 & 13.77 & 96.10 \\
TA-MFC $^{d}$ & 8.79 & 6.21 & 3.15 & 34.40 \\
& & & & \\
Week 6 $_{\text {Control-MFC }}$ & 2.12 & 4.59 & 19.11 & 45.50 \\
Cat-MFC $^{b}$ & 2.72 & 24.12 & 0.85 & 22.80 \\
TV-MFC $^{c}$ & 2.70 & 26.66 & 1.8 & 46.10 \\
TA-MFC $^{d}$ & 5.60 & 11.47 & 1.17 & 40.00
\end{tabular}

${ }^{a}$ Control-MFC: without electrode modification and no inoculum pretreatment. ${ }^{b}$ Cat-MFC: having $\mathrm{V}_{2} \mathrm{O}_{5}$-MF catalysed cathode and GOPTFE modified anode. ${ }^{c}$ TV-MFC: having $\mathrm{V}_{2} \mathrm{O}_{5}$-MF catalysed cathode, GO-PTFE modified anode and anodic biofilm modification with crude extract of $T$. viride. ${ }^{d}$ TA-MFC: with $\mathrm{V}_{2} \mathrm{O}_{5}-\mathrm{MF}$ catalysed cathode, GOPTFE modified anode and anodic biofilm modification with crude extract of T. atroviride. diffusion resistance over six weeks of operation of MFC for TVMFC and TA-MFC can be represented by the Warburg element $\left(W_{\mathrm{d}}\right)$, demonstrating higher power and current output during the polarization study (Fig. 5b). This leads to efficient substrate diffusion through the anodic biofilm due to the enrichment of electro-active microbial species.

\subsection{Wastewater treatment and coulombic efficiencies}

In the stable cycles of operation, the oxidation of organic substrates present in wastewater was achieved by bacterial metabolism. The removal of COD was observed to be $87 \pm 4 \%$, $78 \pm 7 \%, 57 \pm 5 \%$ and $55 \pm 2 \%$ for Control-MFC, Cat-MFC, TVMFC and TA-MFC, respectively, showing a decreasing order of COD removal efficiency; however, the order of power generation was the reverse. The maximum fraction of recovered electrons calculated in terms of CE was 17\%, 27.4\%, 48.7\% and $53.5 \%$ for Control-MFC, Cat-MFC, TV-MFC and TA-MFC, respectively (Fig. 6). Utilization of the available carbon source by methanogenic archaea was comparatively higher in the Control-MFC and Cat-MFC, demonstrating higher COD removal efficiency and lower $\mathrm{CE}$ in the presence of mixed anaerobic sludge as inoculum; whereas, in the cases of TV-MFC and TA-MFC, where peptaibiotics pre-treated inoculum was used, the MFC showed lower COD removal efficiency and higher $\mathrm{CE}$, due to the suppression of the metabolic activity of methanogenic archaea present in the inoculum. The presence of methanogens utilizing acetate as a carbon source (acetoclastic methanogenesis) may significantly lower the coulomb recovery and subsequent generation of electrical power by competing with electrochemically active bacteria for substrate.

The SMA of the MFC inoculum treated with T. viride and $T$. atroviride extract was considerably lower $\left(0.155 \mathrm{~g} \mathrm{CH}_{4}\right.$-COD per $\mathrm{g}$ VSS per day and $0.129 \mathrm{~g} \mathrm{CH}_{4}$-COD per $\mathrm{g}$ VSS per day, respectively) than the SMA of $0.381 \mathrm{~g} \mathrm{CH}_{4}$-COD per $\mathrm{g}$ VSS per day for the inoculum without any sludge pre-treatment. Hence, it can be inferred that the fungi extract can considerably reduce and control the rate of methanogenesis, which has been reflected in the amount of methane generation from Test 1-SMA and Test 2SMA, where the inoculum was pretreated with $T$. viride and $T$. atroviride extract, respectively. This fact was further established

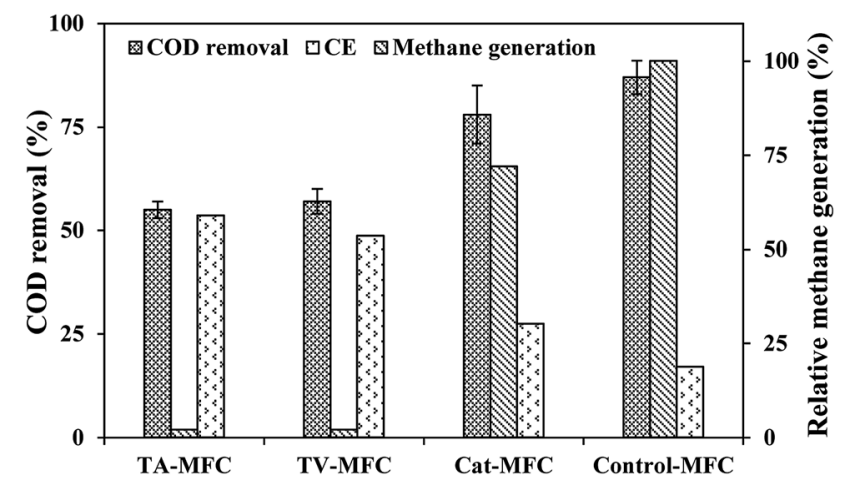

Fig. 6 Wastewater treatment efficiency and coulomb recovery in primary $Y$-axis) and relative methane generation in secondaray $Y$-axis. 
Table 2 Comparative performance of MFCs under methanogen controlling conditions ${ }^{a}$

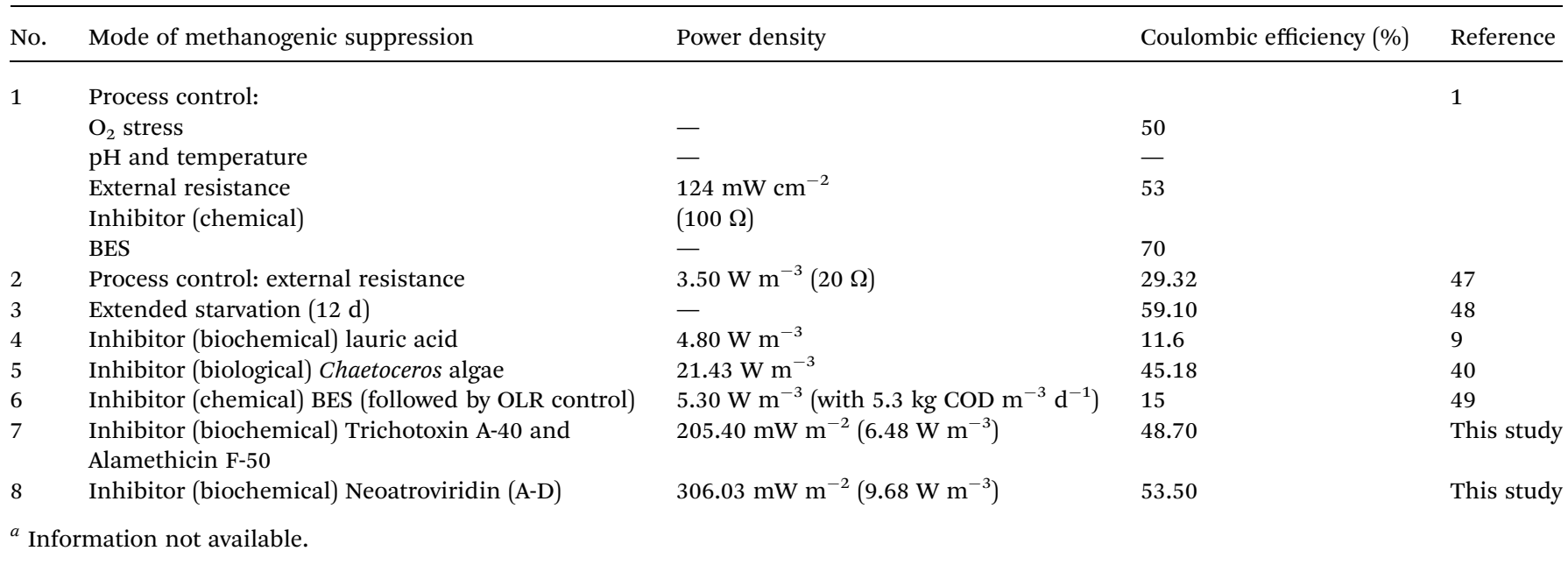

by analysing the head-space biogas samples of MFC, collected in small glass vials sealed with butyl rubber stoppers and Teflon, using GC/TCD. The biogas analysis provided evidence of $24.8 \%$ relative reduction in methane concentration in Cat-MFC, compared to Control-MFC, possibly due to better enriched growth of electrogenic species on the anode surface because of the presence of GO-PTFE, which supported the enhanced electrochemical properties of the anode (ESI, Fig. S5 $\dagger$ ). However, the rate of biogas generation from both TV-MFC and TA-MFC was close to the non-detectable range, due to GO-PTFE modification of the anode surface and effective suppression of the methanogenic activity of anaerobic mixed sludge inoculum by fungal peptaibiotics. Treatment with GO-PTFE may sufficiently increase the specific surface area of the carbon felt anode for effective biofilm formation, thus revealing increased power generation with respect to the untreated anode, i.e., bare carbon felt. Accumulation of non-electrogenic bacteria over the anode surface hinders the active diffusion of analyte. A similar observation was revealed in the EIS study as well, where the decrease of power generation in un-modified MFC happened due to the higher diffusion resistance of the analyte.

Comparative results of this study with relevant findings from other published work are reported in Table $2,,^{1,9,47-49}$ showing the limitations to achieving higher coulomb recovery during longterm operation of MFC. Furthermore, certain drawbacks to using BES as methanogenic suppressant are as follows: firstly, a common agreement on the lethal dose is not yet known, which leads to higher concentrations of unacceptable chemicals in the effluent of MFC; secondly, methane production in MFC is again observed after a certain duration of operation, even after giving a higher BES dose. ${ }^{45}$ It is evident that bio-based materials such as the fungal peptaibiotics are efficient at enhancing the $\mathrm{CE}$ of MFC to support higher power densities to recover more power by improving coulombic efficiency. Long-term use of antibiotics may aid the growth of drug-resistant bacteria and it could severely reduce the electrical performance of MFC due to further growth of methanogens. However, during long-term operation of MFCs for more than eight months under controlled conditions, no severe changes in electrical performances were observed. It was observed that the negative growth effect of surviving methanogens after peptaibiotic pretreatment was minimal. Due to the hydrophobic nature of fungal peptaibiotics, they were not leached; hence, there was no trace of peptaibiotics in the MFC effluent, as confirmed by HPLC analysis.

\subsection{Production of peptaibiotics from Trichoderma sp. - the economic aspect}

The use of economical substrate for fermentation is the only variable cost for the laboratory production of fungal peptaibiotics. The extraction and partial purification process comprises the separation of mycelia from broth, washing with clean water and solid phase two-solvent extraction. Organic solvents are recyclable and hence it makes the down-stream process economically viable. Considering the cost of fungal fermentation and recovery of peptaibiotics as $44 \%$ of the total manufacturing cost (including variable cost, utilities, strain management and fixed cost), ${ }^{46}$ the extracts recovered from $T$. viride and T. atroviride were priced at $0.48 \$$ per $\mathrm{g}$ and $1.32 \$$ per $\mathrm{g}$, respectively. The cost of T. viride extract, dominated by Trichotoxin A-40 and Alamethicin F-50, is 120 fold lower than the price of commercially available Alamethicin. Other peptaibiotics from Trichoderma sp. were not available commercially. Hence, this study is offering the prospect of more economical production of fungal peptaibiotics and their use in anodic inoculum pre-treatment for enriching electrogens in the anodic chamber of MFC to increase the power generation several fold.

\section{Conclusion}

Loss of electrons due to bio-methanation is one of the critical issues in MFC research dealing with mixed microbial consortia. Membrane-active peptaibiotics cause cytolysis in Gram-positive bacteria. However, upon application of methanogenic suppression in mixed anaerobic sewage sludge used as anodic inoculum, MFC treated with Neoatroviridin (A-D), extracted 
from Trichoderma atroviride, demonstrated the highest volumetric power density of $9.68 \mathrm{~W} \mathrm{~m}^{-3}$ and current density of 31.89 $\mathrm{A} \mathrm{m}^{-3}$ with highest $\mathrm{CE}$ of $53.5 \%$. In this study, almost complete inhibition of methanogenic archaea was achieved by inoculum pre-treatment using low-cost peptaibiotics, leading to a longterm, attractive and promising way to achieve higher range of coulomb retrieval and improved power generation from MFC.

\section{Acknowledgements}

This work was supported by Ministry of New and Renewable Energy (REF, Ministry sanction letter no. 10/14/2010) giving fellowship to the first author and Department of Science of Technology, New Delhi, Government of India (DST/INT/UK/P101/2014) for providing funding for this research.

\section{References}

1 K. J. Chae, M. J. Choi, K. Y. Kim, F. F. Ajayi, W. Park, C.-W. Kim and I. S. Kim, Bioresour. Technol., 2010a, 101, 5350-5357.

2 S. Freguia, K. Rabaey, Z. Yuan and J. Keller, Environ. Sci. Technol., 2007, 41, 2915-2921.

3 H. Liu, J. Wang, A. Wang and J. Chen, Appl. Microbiol. Biotechnol., 2011, 89, 1333-1340.

4 B. R. Tiwari and M. M. Ghangrekar, Energy Fuels, 2015, 29, 3518-3524.

5 Y. Fan, H. Hu and H. Liu, J. Power Sources, 2007, 171, 348354.

6 K. J. Chae, M. J. Choi, K. Y. Kim, F. F. Ajayi, I. S. Chang and I. S. Kim, Int. J. Hydrogen Energy, 2010b, 35, 13379-13386.

7 A. E. West and S. K. Schmidt, Microb. Ecol., 2002, 43, 408415.

8 X. Zhu, M. Siegert, M. D. Yates and B. E. Logan, Appl. Environ. Microbiol., 2015, 81, 3863-3868.

9 P. P. Rajesh, M. T. Noori and M. M. Ghangrekar, Water Sci. Technol., 2014, 70, 1363-1369.

10 J. R. Kim, B. Min and B. E. Logan, Appl. Microbiol. Biotechnol., 2005, 68, 23-30.

11 T. Catal, K. L. Lesnik and H. Liu, Bioresour. Technol., 2015, 187, 77-83.

12 L. Wang, Y. Wu, Y. Zheng, L. Liu and F. Zhao, RSC Adv., 2015, 5, 56430-56437.

13 L. Wang, Y. Liu, J. Ma and F. Zhao, Water Res., 2016, 88, 322328.

14 C. P. Kubicek, M. Komoń-Zelazowska, E. Sándor and I. S. Druzhinina, Chem. Biodiversity, 2007, 4, 1068-1082.

15 S. Zeilinger, Fungal Biomolecules: Sources, Applications and Recent Developments, John Wiley \& Sons Ltd., Chichester, UK, 2015.

16 J. K. Chugh and B. A. Wallace, Biochem. Soc. Trans., 2001, 29, 565-570.

17 H. Duclohier and H. Wroblewski, J. Membr. Biol., 2001, 184, $1-12$.

18 M. Dathe, C. Kaduk, E. Tachikawa, M. F. Melzig, H. Wenschuh and M. Bienert, Biochim. Biophys. Acta, Biomembr., 1998, 1370, 175-183.
19 C. Schwechheimer and M. J. Kuehn, Nat. Rev. Microbiol., 2015, 13, 605-619.

20 D. P. Tieleman, H. J. Berendsen and M. S. Sansom, Biophys. J., 1999, 76, 1757-1769.

21 S. P. Denyer and J. Y. Millard, J. Appl. Microbiol., 2002, 92, 3545.

22 R. Benz and K. Bauer, Eur. J. Biochem., 1988, 176, 1-19.

23 A. H. Delcour, Biochim. Biophys. Acta, Proteins Proteomics, 2009, 1794, 808-816.

24 The Biochemistry of Archaea, ed. M. Kates, D. J. Kushner and A. T. Matheson, Elsevier, New York, USA, 1993.

25 B. Demirel and P. Scherer, Rev. Environ. Sci. Bio/Technol., 2008, 7, 173-190.

26 R. Casadonte and R. M. Caprioli, Nat. Protoc., 2011, 6, 16951709.

27 N. Stoppacher, N. K. Neumann, L. Burgstaller, S. Zeilinger, T. Degenkolb, H. Brückner and R. Schuhmacher, Chem. Biodiversity, 2013, 10, 734-743.

28 I. W. Koster and A. Cramer, Appl. Environ. Microbiol., 1987, 53, 403-409.

29 W. S. Hummers Jr and R. E. Offeman, J. Am. Chem. Soc., 1958, 80, 1339.

30 M. T. Noori, M. M. Ghangrekar and C. K. Mukherjee, Int. J. Hydrogen Energy, 2016, 41, 3638-3645.

31 APHA, Standard methods for examination of water and wastewater, American Public Health Association, American Water Works Association, Water Environment Federation, Washington DC, USA, 20th edn, 1998.

32 A. Pandey, P. Sinha, S. M. Kotay and D. Das, Int. J. Hydrogen Energy, 2009, 34, 7483-7488.

33 K. Fricke, F. Harnisch and U. Schröder, Energy Environ. Sci., 2008, 1, 144-147.

34 J. Liu, Y. Qiao, C. X. Guo, S. Lim, H. Song and C. M. Li, Bioresour. Technol., 2012, 114, 275-280.

35 K. Rabaey, N. Boon, S. D. Siciliano, M. Verhaege and W. Verstraete, Appl. Environ. Microbiol., 2004, 70, 5373-5382.

36 H. S. Park, B. H. Kim, H. S. Kim, H. J. Kim, G. T. Kim, M. Kim, I. S. Chang, Y. K. Park and H. I. Chang, Anaerobe, 2001, 7, 297-306.

37 C. R. Röhrich, A. Vilcinskas, H. Brückner and T. Degenkolb, Chem. Biodiversity, 2013, 10, 827-837.

38 J. Kirschbaum, C. Krause, R. K. Winzheimer and H. Brückner, J. Pept. Sci., 2003, 9, 799-809.

39 S. U. Oh, B. S. Yun, S. J. Lee and I. D. Yoo, Appl. Microbiol. Biotechnol., 2005, 15, 384-387.

40 P. P. Rajesh, D. A. Jadhav and M. M. Ghangrekar, Bioresour. Technol., 2015, 180, 66-71.

41 Y. Fan, E. Sharbrough and H. Liu, Environ. Sci. Technol., 2008, 42, 8101-8107.

42 E. C. Salas, Z. Sun, A. Luttge and J. M. Tour, ACS Nano, 2010, 4, 4852-4856.

43 S. Khilari, S. Pandit, M. M. Ghangrekar, D. Das and D. Pradhan, RSC Adv., 2013, 3, 7902-7911.

44 N. Sekar and R. P. Ramasamy, J. Microb. Biochem. Technol., 2013, S6, 004. 
45 B. S. Guri, F. M. L. Vega, J. Cirucci, P. N. Montpart, S. F. J. Lafuente, L. J. A. Baeza and C. A. Guisasola, Pat. EP2747181 A1, 25 Jun 2014.

46 A. J. Hacking, Economic Aspects of Biotechnology, Cambridge University Press, Cambridge, 1986.

47 H. Rismani-Yazdi, S. M. Carver, A. D. Christy, Z. Yu, K. Bibby, J. Peccia and O. H. Tuovinen, Bioresour. Technol., 2013, 129, 281-288.
48 A. Kaur, H. C. Boghani, I. Michie, R. M. Dinsdale, A. J. Guwy and G. C. Premier, Bioresour. Technol., 2014, 173, 75-81.

49 D. Molognoni, S. Puig, M. D. Balaguer, A. G. Capodaglio, A. Callegari and J. Colprim, J. Chem. Technol. Biotechnol., 2016, 91, 1720-1727. 